\title{
Avalanche Statistics Identify Intrinsic Stellar Processes near Criticality in KIC 8462852
}

\author{
Mohammed A. Sheikh, Richard L. Weaver, and Karin A. Dahmen \\ Department of Physics, 1110 West Green Street, University of Illinois at Urbana Champaign, Urbana, Illinois 61801, USA
}

(Received 12 July 2016; published 19 December 2016)

\begin{abstract}
The star KIC8462852 (Tabby's star) has shown anomalous drops in light flux. We perform a statistical analysis of the more numerous smaller dimming events by using methods found useful for avalanches in ferromagnetism and plastic flow. Scaling exponents for avalanche statistics and temporal profiles of the flux during the dimming events are close to mean field predictions. Scaling collapses suggest that this star may be near a nonequilibrium critical point. The large events are interpreted as avalanches marked by modified dynamics, limited by the system size, and not within the scaling regime.
\end{abstract}

DOI: 10.1103/PhysRevLett.117.261101

The Kepler spacecraft was conceived with the goal of finding extrasolar planets by measuring the time-varying light flux (defined as power received in the $423-897 \mathrm{~nm}$ wavelength range [1]) from about 150000 stars [1-3]. Beyond that original goal, the light curves (flux vs time) are now used for other purposes [4]. A dramatic example of such an application is the investigation of anomalies associated with the star KIC 8462852. By most metrics, KIC 8462852 is a normal F-class star. However, the Kepler data has shown drops in flux by more than $20 \%$, as well as irregular smaller variations in flux in excess of similar fluctuations in many other stars [5]. The unique characteristics of this light curve have motivated much interest in KIC 8462852. Here we point out that these fluctuations are reminiscent of avalanches. We show that the statistics of the fluctuations are similar to those often seen in ferromagnets and plastic flow and to predictions from a mean field theory $[6,7]$.

Avalanches in this context are intermittent, sudden changes in a system under a slowly increasing driving force [8]. One example is provided by the nucleation and growth of magnetic domains in ferromagnets in a changing magnetic field. The spins in a ferromagnet are originally taken to be randomly oriented, but as an external magnetic field is increased, the spins tend to align to the applied field. Spin-spin interactions couple the spins with each other, and as one spin flips, coupled spins may also; the effect can cascade and become an avalanche of spin flips. A small but discrete jump in the total magnetization results. This behavior is known as Barkhausen noise [9]. Many systems exhibit similar crackling noise that can be explained as avalanches $[10,11]$. This noise is often characterized by several power law probability distributions. In astrophysical systems, avalanche statistics have been found to describe the distribution of energies of $x$-ray emission from stellar flares [12], $\mathrm{x}$ rays seen from soft $\gamma$-ray repeaters [13], and a variety of other phenomena [14].

For avalanches, detail independent (universal) properties are often found in the size and duration distributions and their associated power law exponents [15]. These distributions follow universal power laws that are multiplied by cutoff functions. The regime over which the power law holds is known as the scaling regime. A wide scaling regime indicates that the system has avalanches that span a broad range of sizes and durations. In some systems, very large avalanches sensitive to the system size can also occur. These avalanches are characterized as large earthquakelike events $[6,16]$ that have different statistics and modified dynamics from small avalanches. Motivated by the large dimming events in KIC 8462852, we have analyzed also the small dimming events (those on the order of $10^{-5}-10^{-3}$ of the median value) and compared their statistics to those of avalanches in magnets and plastic flow.

An avalanche is identified as a dimming event in the light curve that drops below a chosen threshold. We choose the threshold to be the median value of the light curve [17]. In Fig. 1 we show the original light curve and an example of two avalanches. The entire four-and-a-half-year light curve yields approximately 1000 avalanches in the scaling regime, but the precise number depends on the choice of threshold. The duration $T$ of an avalanche is the time the light curve remains below the threshold [Fig. 1(b)]. The size $S$ of an avalanche is defined as the time integral of the flux for the duration of the dimming event, in analogy with Barkhausen noise experiments [10]. Interpreted another way, the time integral of the flux is proportional to the net loss of radiant fluence (radiant energy per unit area), in analogy with the statistics extracted from soft $\gamma$-ray repeaters [13] or stellar flares [18]. In order to remove unrelated sources of fluctuations from the data, the 0.88day rotation period of the star and its two higher harmonics are filtered out using a notch filter (see Supplemental Material [19]).

A simple avalanche model predicts the universal scaling exponents for the avalanche statistics and the dynamics. To extract the corresponding power law exponents from the observational data we fit power laws to complementary cumulative distribution functions (CCDFs) of avalanche sizes and durations [19]. The $\operatorname{CCDF} C(S)$ gives the 

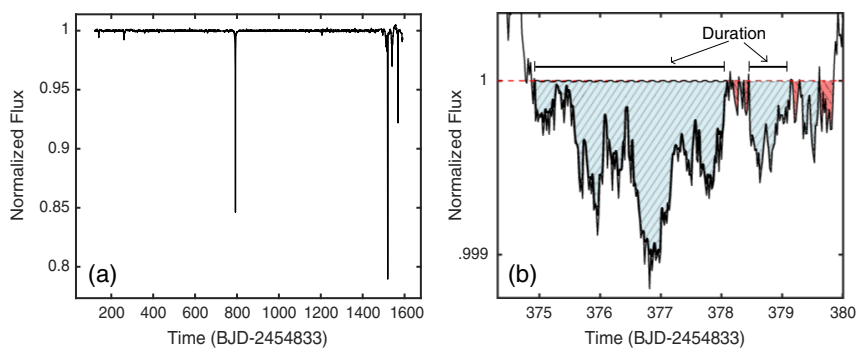

FIG. 1. (a) The light curve (flux vs time) of KIC 8462852 normalized to the median value. (b) An example of how the avalanches were extracted. The threshold (dashed line) was set at the median value, which is normalized to 1 . Different avalanches are denoted by alternating colors and cross-hatch patterns. The duration of two example avalanches is marked by solid intervals. The area underneath is defined as the size of the avalanche.

probability of obtaining an avalanche of size larger than $S$. Additional tests of the model are extracted through scaling collapses of avalanche time profiles. Table I summarizes the obtained exponent values.

Figure 2 shows a remarkable power law behavior of avalanche size distributions in KIC 8462852, spanning nearly two decades of probability. We summarize the predictions for the avalanche statistics $[6,15,16]$ and the various tests of consistency between the KIC 8462852 events and the avalanche model. We consider three exponents $\tau, \alpha$, and $\sigma \nu z$. The exponent $\tau$ is defined as the exponent of the CCDF $C(S) \sim S^{-\tau+1}$, where the size $S$ is in the scaling regime $S_{\min }<S<S_{\max }$ [Fig. 2(a)]. The CCDF $C(T) \sim T^{-\alpha+1}$ defines $\alpha$ for an avalanche of duration $T$ in the regime $T_{\min }<T<T_{\max }$ [Fig. 2(b)]. There is also a power law relationship between $S$ and $T$ given by $T \sim S^{\sigma \nu z}$, defining $\sigma \nu z$ [Fig. 2(c)]. The exponent $\sigma \nu z$ is the product of three exponents commonly defined in avalanche and crackling noise literature [10]. The power spectral density, the magnitude squared of the Fourier transform of the light curve, is expected to follow $P(\omega) \sim \omega^{-1 / \sigma \nu z}$ [15] with

TABLE I. The exponent values for KIC 8462852 and the mean field avalanche model. In order to extract the exponents $\tau$ and $\alpha$ and find uncertainties, we used a maximum likelihood approach [31]. The uncertainties obtained from the variation of threshold and variation of the scaling regime were much larger than that from bootstrapping, so we used these uncertainty values in the Table (see Supplemental Material [19]). The actual error bars may be larger; the maximum likelihood method we have implemented does not take into account the decaying cutoff function [32].

\begin{tabular}{lcc}
\hline \hline Exponent & KIC 8462852 exponents & Mean field value \\
\hline$\tau$ & $1.60 \pm_{0.13}^{0.10}$ & $3 / 2$ \\
$\alpha$ & $2.09 \pm_{0.40}^{0.07}$ & 2 \\
$\sigma \nu z$ & $0.67 \pm_{0.02}^{0.04}$ & $1 / 2$ \\
$(\tau-1) /(\alpha-1)$ & $0.55 \pm_{0.14}^{0.46}$ & $1 / 2$ \\
\hline \hline
\end{tabular}

$\omega_{\min }=1 / T_{\max }$ and $\omega_{\max }=1 / T_{\min }$ [Fig. 2(d)]. The power spectral density is plotted after removal of the very large avalanches, and after filtering out the rotation signal. The filtered frequencies may be seen in the notches seen in the power spectral density in Fig. 2(d). The scaling regimes are highlighted in red in Fig. 2. The scaling regimes are found using scaling collapses, as defined below (see Supplemental Material [19]). One test of consistency with theoretical predictions [15] is the exponent relation $(\tau-1) /(\alpha-1)=\sigma \nu z$. This is satisfied by size and duration distributions of KIC 8462852 to within error bars.

The statistics of these small dimming events are similar to those of an avalanche model near a nonequilibrium phase transition [33]. The exponents for KIC 8462852 and the mean field avalanche model [6,7] are listed in Table I. These exponents were extracted using the maximumlikelihood criteria [31]. The associated uncertainties were estimated by varying both the threshold and the scaling regime and recalculating the avalanche statistics. The threshold was varied between $\pm 10^{-3} \%$ (which is large given our avalanche size), and our identification of the scaling regime was varied by $\pm 64 \%$ (see Supplemental Material [19] for details).
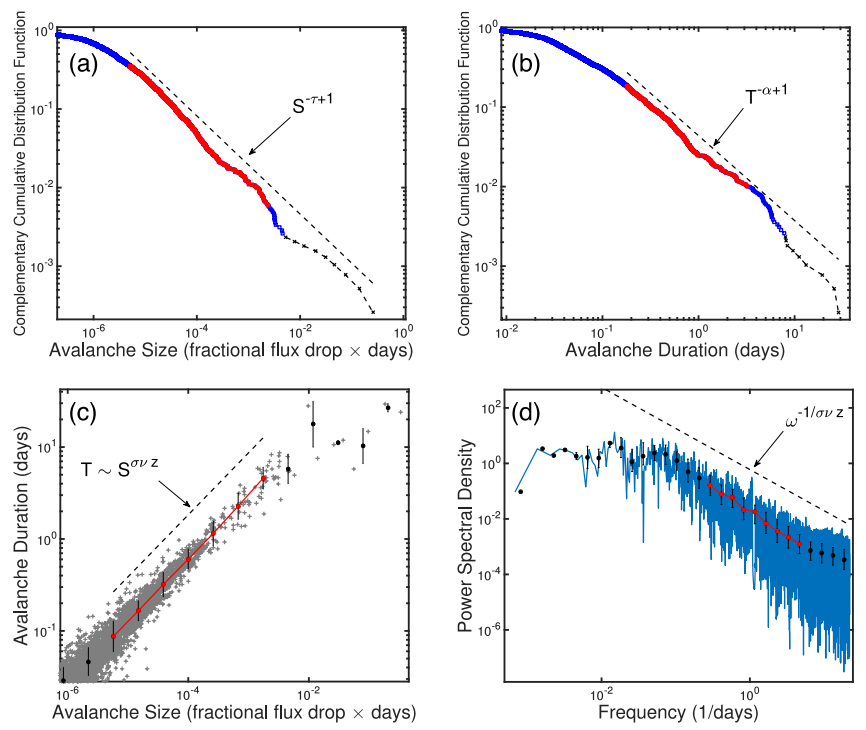

FIG. 2. Log-log plots of the various avalanche statistics. The highlighted (red) region denotes the scaling regime (a)-(d) (see Supplemental Material [19]). In (a) and (b) the black crosses represent the 9 largest avalanches. In (a),(b), and (c) the dashed line denotes the power law with the measured exponent (Table I). (a) The CCDF of avalanche sizes. (b) The CCDF of avalanche durations. (c) The avalanche magnitude vs duration. The dots denote logarithmic bins in size. (d) The power spectral density. Here the dashed line is the power law with exponent -1.5 , which matches well with the predicted value of the slope $1 / \sigma \nu z$ [15], where $\sigma \nu z$ was defined from the slope of (c). Incidentally, the slope appears to cross over at frequencies below the scaling regime (below about $0.31 /$ days) to $1 / \sigma \nu z=2$, which is the mean field value. 
To further test the model, we look at the shapes of the dimming events [34]. The Kepler light curves are composed of data points taken every $30 \mathrm{~min}$. This high temporal resolution relative to the duration of the avalanches (on the order of hours to days) allows us to observe detailed features of even the small dimming events. This gives us a powerful test to confirm that the dimming events in KIC 8462852 are similar to the mean field avalanche model near criticality [6]. The theory of critical phenomena posits that near criticality the average temporal avalanche profile will follow a universal shape as a function of time [35]. This universal profile is not visible in a single avalanche because an avalanche is an inherently noisy process. However, the data averaged in a systematic way should exhibit a universal profile. The averages are plotted in Fig. 3 after rescaling to show the universal profiles.

There are two types of averages that can be performed to find different universal profiles. The first is an average over avalanches of a specified duration. Here, the average is denoted $\langle V(t) \mid T\rangle=T^{1 / \sigma \nu z-1} f(t / T)$, where $f(t / T)$ is the universal shape of the average, $T$ is the duration, and $V(t)$ is the temporal profile of an avalanche. In practice, very few avalanches have the same duration, so we average over avalanches between $0.85 T<T<1.15 T$ for logarithmically spaced values of $T$. The avalanches are first interpolated to a fixed number of time points after which they are averaged. The scaling collapse of the avalanches is then performed by dividing the averaged shapes by the factor $T^{1 / \sigma \nu z-1}$ and rescaling the time axis by $T$. In Fig. 3(a), the inset shows the original averaged shapes and the larger plot shows the scaling collapse. The scaling collapse uses the measured value of $\sigma \nu z=0.67$, but collapses within the
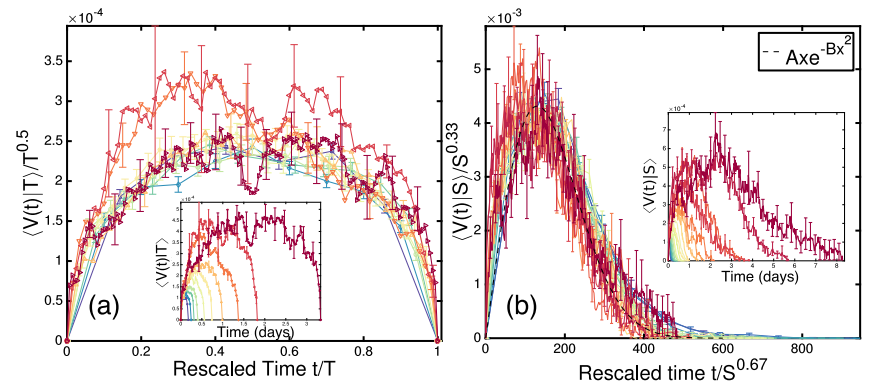

FIG. 3. The collapses of avalanches after averaging over duration (a) and size (b). We plot every 10th error bar to prevent cluttering. The insets show the original averages before rescaling. The data is inverted from the original Kepler photometry data (i.e., positive numbers here mean a larger drop from the threshold). Redder colors correspond to longer avalanches, while bluer colors correspond to shorter avalanches. The value of the factor $\sigma \nu z$ was taken to be 0.67 , the measured value. In (a) the rescaled time is $t / T$, where $T$ is the total duration. The scaling factor $T^{0.5}=T^{1 / \sigma \nu z-1}$. In (b) the dashed black line shows the best fit mean field theory prediction for the collapsed function. The rescaled time is $t / S^{0.67}=t / S^{\sigma \nu z}$ and the scaling factor $S^{0.33}=S^{1-\sigma \nu z}$. range $0.60<\sigma \nu z<0.71$ were reasonable. We have also used this scaling collapse to define the scaling regime as the range of durations for which the avalanche shapes can be averaged and collapsed successfully.

A natural second type of averaging involves an average over avalanches of the same size [6]. To average over avalanches of the same size, the avalanches are zero-padded to the maximum duration in a bin. Again we average over avalanches between $0.85 S<S<1.15 S$ for logarithmically spaced values of $S$. In [Fig. 3(b)], the inset shows the averaged shapes and the larger plot shows the collapsed average, with the value of $\sigma \nu z=0.67$. Collapses within the range $0.60<\sigma \nu z<0.71$ were reasonable. We see that the averaged shapes collapse onto a single curve. The dashed black line is the mean field prediction for this curve, which follows the collapsed avalanches well. Additionally, the collapse allows us to determine a scaling regime for avalanche sizes.

We have analyzed the light curves of several other Kepler stars (mostly F class) to compare with KIC 8462852. We have not systematically explored all stars monitored by Kepler. Three stars, KIC 4638884, KIC 7771282 [36], and KIC 5955122 [37], were found to have scaling collapses and statistics that match the avalanche model predictions (see Supplemental Material [19]). The main feature of these three stars is the presence of magnetic activity, as previously characterized $[4,36,37]$ by the spectrum of their light curve data. We remark that most of the stars we analyzed do not exhibit evidence for avalanches.

In Fig. 4 we have plotted the extracted CCDFs of the avalanche sizes of KIC 8462852 and the three magnetically active stars. If the stars are near an underlying critical point, then universality predicts that these CCDFs should have the same functional scaling form. The cutoffs of the scaling regime will vary due to differing values of one (or more) tuning parameters [9] describing differing distances to the underlying critical point. We plot this in the inset of Fig. 4. To perform the CCDF collapse, the second moment of the avalanche sizes was calculated for each of the stars after excluding the Gaussian noise regime of sizes $\left(10^{-10}-10^{-6}\right)$. The second moment was then used as a proxy for the cutoff size of the scaling regime, with $S_{\text {cutoff }} \sim\left\langle S^{2}\right\rangle^{3-\tau}$ [11]. The CCDFs were then collapsed using the mean field exponent $\tau=1.5$ [11]. Values of $\tau$ that produce acceptable collapses are between $1.3<\tau<1.6$. Note that this range includes the range of values of $\tau$ for each of the four stars (see Supplemental Material [19]). The 9 largest avalanches in KIC 8462852 were removed prior to finding the moments because these avalanches are believed to follow modified and system-size dependent dynamics compared to the smaller avalanches. The variations in the cutoff imply that the small avalanches in KIC 8462852 are not due a process described by self-organized criticality, but rather to tuned criticality. Examples of similar tuned criticality occurs in ferromagnetic systems, where the tuning parameter is the 


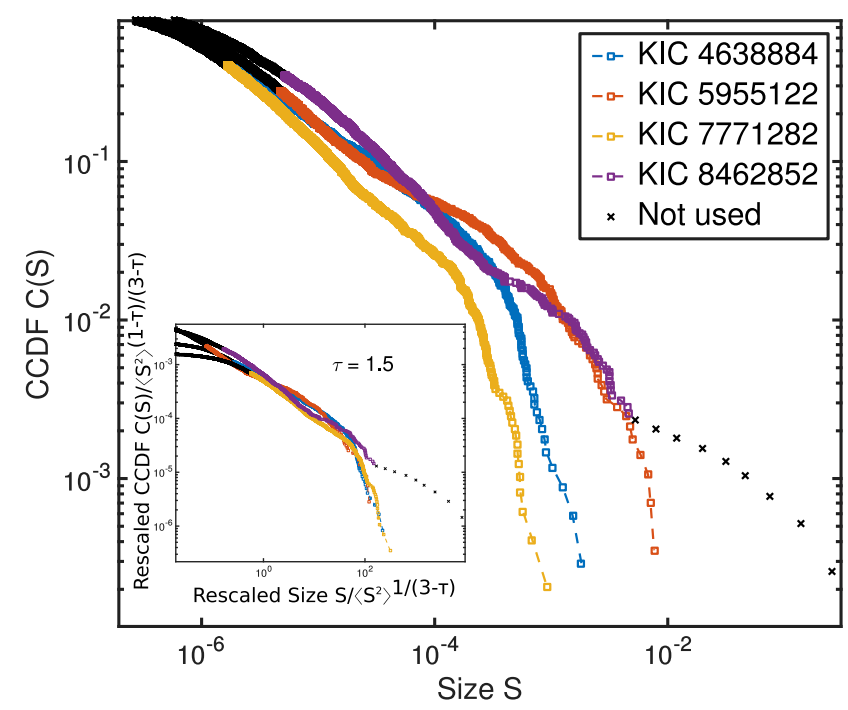

FIG. 4. The size CCDFs of the four stars labeled in the figure. Data points marked with squares were used in the calculation of the moments. Data points marked with black crosses were not included in the calculation of the moments. The scaling regime of KIC 8462852 is between $5 \times 10^{-6}$ and $2.4 \times 10^{-3}$ in size. The other stars have similar scaling regimes, approximately between $3 \times 10^{-6}$ and $1 \times 10^{-4}$. In the inset the rescaled CCDFs were plotted to show that there is a possible relationship between these four stars. We chose the mean field exponent $\tau=1.5$ in the collapse, but values between $1.3<\tau<1.6$ produce reasonable results. In KIC 8462852, the 9 largest avalanches were truncated from the calculation of the moment.

external magnetic field [10], and plastic deformation of solids, where the tuning parameter is applied stress [6]. We do not conjecture what the tuning parameters are for the avalanches in KIC 8462852.

We have analyzed the small dimming events in the light curve of KIC 8462852 , but it was the very large dimming events that drew attention to the star. Based on the data, the small dimming events in the light curve follow predictions close to the mean field model. The large avalanches are too few to permit statistical analysis. However, motivated by the mean field model we can speculate about the nature of both the small and large events. The very large avalanches are similar to large avalanches in plasticity, where finite size or weakening effects cause large avalanches to deviate from power law scaling. The weakening parameter determines the nucleation size for large events that span the system [6]. Large slip avalanches, similar to the large dimming events in KIC 8462852, can be seen in the plastic deformation of bulk-metallic glass [38] (see Supplemental Material [19]).

The broad range over which the power laws apply to the avalanche statistics in KIC 8462852 implies that there are intrinsic stellar processes near criticality. The large dimming events seen in the light curve could also be due to an intrinsic stellar process rather than occultation of the star by some external object. In particular, we believe KIC 8462852 is a type of variable star, whose intrinsic stellar variability is perhaps related to the magnetic activity of the star.

In many other stars, stellar variability following power law behavior has been related to regions of intense stellar magnetic activity $[39,40]$ or stars with very large magnetic fields [13]. We do not claim that these mechanisms are responsible for the activity seen in KIC 8462852. We only point out that there is a surprising agreement between the exponents reported in this letter and those reported for stellar flares [12,18,39-41] and soft $\gamma$-ray repeaters [13]. Several superflare studies have been done using the Kepler data, with the exponent analogous to $\tau$ in this report of $1.6-2.3$ [18,39-42], $\alpha \sim 2$, and $\sigma \nu z \sim .5-1[43,44]$. The value of the exponent of avalanche size $\tau=1.60$ is also close to the exponent of 1.67 of distribution of the energies of bursts of $\mathrm{x}$ rays from soft $\gamma$-ray repeaters [13].

If the dimming events seen in KIC 8462852 are a result of intrinsic phenomena similar to the mean field model avalanche model [6], then there are probably tuning parameters that can move the system towards a nonequilibrium phase transition. This would explain the rarity of a light curve like that of KIC 8462852 . If KIC 8462852 is near a nonequilibrium phase transition, this could also explain the random times at which the large events occur in the light curve [5]. However, there is more work to be done in order to verify that KIC 8462852 is near a critical point. If intrinsic stellar variability of KIC 8462852 is responsible for the large dimming events, then a detailed theory of stellar processes is necessary to answer what the key tuning parameters are. A theory to explain the power law distribution of the small dimming events could help in uncovering the mystery of the large events. Even in the case that the large dimming events are not related to the small dimming events, the statistics of these small events need to be explained. There is also the possibility that many other stars may have interesting avalanche statistics. We have shown several tools that can be used to compare and categorize stars based on these statistics.

We acknowledge support from the U.S. National Science Foundation (NSF) Grants No. DMR 10-05209, No. CBET 1336634 (K. D.). We thank Lars Bildsten, Michael LeBlanc, Will McFaul, Li Shu, and Jonathan T. Uhl for helpful conversations. K. D. thanks the KITP for hospitality and support through NSF Grant No. PHY-1125915.

*masheik2@illinois.edu

dahmen@illinois.edu

[1] W. J. Borucki et al., Science 327, 977 (2010).

[2] A. S. Baran, S. Zola, A. Blokesz, R. H. Østensen, and R. Silvotti, Astron. Astrophys. 577, A146 (2015).

[3] G. Basri et al., Astrophys. J. 713, L155 (2010).

[4] W. J. Chaplin et al., Science 332, 213 (2011).

[5] T. S. Boyajian et al., Mon. Not. R. Astron. Soc. 457, 3988 (2016). 
[6] K. A. Dahmen, Y. Ben-Zion, and J. T. Uhl, Phys. Rev. Lett. 102, 175501 (2009).

[7] K. A. Dahmen, Y. Ben-Zion, and J. T. Uhl, Nat. Phys. 7, 554 (2011).

[8] J. P. Sethna, K. Dahmen, S. Kartha, J. A. Krumhansl, B. W. Roberts, and J. D. Shore, Phys. Rev. Lett. 70, 3347 (1993).

[9] S. Zapperi, P. Cizeau, G. Durin, and H. E. Stanley, Phys. Rev. B 58, 6353 (1998).

[10] J. P. Sethna, K. A. Dahmen, and C. R. Myers, Nature (London) 410, 242 (2001).

[11] J. T. Uhl et al. Sci. Rep. 5, 16493 (2015).

[12] E. T. Lu and R. J. Hamilton, Astrophys. J. Lett. 380, L89 (1991).

[13] E. Göğüş, P. M. Woods, C. Kouveliotou, J. van Paradijs, M. S. Briggs, R. C. Duncan, and C. Thompson, Astrophys. J. 532, L121 (2000).

[14] M. J. Aschwanden et al., Space Sci. Rev. 198, 47 (2016).

[15] M. C. Kuntz and J. P. Sethna, Phys. Rev. B 62, 11699 (2000).

[16] D. S. Fisher, K. Dahmen, S. Ramanathan, and Y. Ben-Zion, Phys. Rev. Lett. 78, 4885 (1997).

[17] Although the precise values of the exponents change with varying threshold, the change is not dramatic (see Supplemental Material [19]). The threshold need only be set so that it does not exclude the majority events in the light curves.

[18] H. Maehara, T. Shibayama, S. Notsu, Y. Notsu, T. Nagao, S. Kusaba, S. Honda, D. Nogami, and K. Shibata, Nature (London) 485, 478 (2012)..

[19] See Supplemental Material at http://link.aps.org/ supplemental/10.1103/PhysRevLett.117.261101, for detail about the data processing steps, the three other stars in Fig. 4, and the bulk-metallic glass avalanches, which includes Refs. [20-30].

[20] https://archive.stsci.edu/.

[21] J. L. Christiansen et al., Publ. Astron. Soc. Pac. 124, 1279 (2012).

[22] R. Handberg and M. N. Lund, Mon. Not. R. Astron. Soc. 445, 2698 (2014).

[23] J. E. Van Cleve and D. A. Caldwell, Kepler Instrument Handbook (KSCI-19033-002).

[24] S. J. Murphy, Investigating the A-Type Stars Using Kepler Data (Springer, New York, 2014).
[25] K. Kinemuchi, T. Barclay, M. Fanelli, J. Pepper, M. Still, and S. B. Howell, Astron Soc Pac. 124, 963 (2012).

[26] R. A. García et al., Astron. Astrophys. 568, A10 (2014).

[27] Thomas W. Parks and C. Sidney Burrus, Digital Filter Design. (John Wiley \& Sons, New York, 1987), p. 54.

[28] N. Friendman, Ph.D. Thesis, University of Illinois at Urbana-Champaign, 2013.

[29] B. Efron and R. J. Tibshirani, An Introduction to the Bootstrap (CRC Press, Boca Raton, 1994).

[30] W. J. Wright, R. R. Byer, and X. Gu, Appl. Phys. Lett. 102, 241920 (2013).

[31] A. Clauset, C. Shalizi, and M. Newman, SIAM Rev. 51, 661 (2009).

[32] O. Perković, K. A. Dahmen, and J. P. Sethna, Phys. Rev. B 59, 6106 (1999).

[33] D. S. Fisher, Phys. Rep. 301, 113 (1998).

[34] S. Papanikolaou, F. Bohn, R. L. Sommer, G. Durin, S. Zapperi, and J. P. Sethna, Nat. Phys. 7, 316 (2011).

[35] A. Baldassarri, F. Colaiori, and C. Castellano, Phys. Rev. Lett. 90, 060601 (2003).

[36] S. Mathur, R. A. García, J. Ballot, T. Ceillier, D. Salabert, T. S. Metcalfe, C. Régulo, A. Jiménez, and S. Bloemen, Astron. Astrophys. 562, A124 (2014).

[37] A. Bonanno, H.-E. Fröhlich, C. Karoff, M. N. Lund, E. Corsaro, and A. Frasca, Astron. Astrophys. 569, A113 (2014).

[38] J. Antonaglia, W. J. Wright, X. Gu, R. R. Byer, T. C. Hufnagel, M. LeBlanc, J. T. Uhl, and K. A. Dahmen, Phys. Rev. Lett. 112, 155501 (2014).

[39] B. E. Schaefer, J. R. King, and C. P. Deliyannis, Astrophys. J. 529, 1026 (2000).

[40] H. He, H. Wang, and D. Yun, Astrophys. J. Suppl. Ser. 221, 18 (2015).

[41] C.-J. Wu, W.-H. Ip, and L.-C. Huang, Astrophys. J. 798, 92 (2015).

[42] T. Shibayama, H. Maehara, S. Notsu, Y. Notsu, T. Nagao, S. Honda, T. T. Ishii, D. Nogami, and K. Shibata, Astrophys. J. Suppl. Ser. 209, 5 (2013).

[43] S. L. Hawley, J. R. A. Davenport, A. F. Kowalski, J. P. Wisniewski, L. Hebb, Russell Deitrick, and E. J. Hilton, Astrophys. J. 797, 121 (2014).

[44] J. R. A. Davenport et al., Astrophys. J. 797, 122 (2014). 